\title{
Local Control of Leg Movements and Motor Patterns during Grooming in Locusts
}

\author{
Ari Berkowitz and Gilles Laurent \\ Division of Biology, California Institute of Technology, Pasadena, California 91125
}

This study demonstrates that the thoracic and abdominal nervous system of locusts is sufficient to mediate several sitespecific and distinct grooming leg movements. Locusts can use a hindleg or middle leg to groom at least four ipsilateral thoracic and abdominal sites, without input from the brain, subesophageal ganglion, or prothoracic ganglion. The hindleg is used to groom the posterior abdomen, the ventral or posterior hindleg coxa, and the ear; the middle leg is used to groom the anterior hindleg coxa. Grooming movements are often rhythmic and display site-specific intralimb coordination patterns. During grooming of the posterior abdomen or ventral hindleg coxa, for example, hindleg tibial extension occurs nearly simultaneously with femur elevation, in contrast with locust hindleg movements during walking. Electromyographic (EMG) recordings during these movements show that rhythmic bursts of tibial extensor activity occur nearly in-phase with those of trochanteral leva- tors, in contrast to hindleg EMGs during walking. During grooming of the ear, hindleg tibial extension/flexion and tibial extensor/flexor muscle bursts can occur independently of the femur elevation/depression and trochanteral levator/depressor muscle bursts, suggesting that the neural modules controlling tibial and femoral movements can be uncoupled during this behavior. Tibial extension can occur before, or even in the absence of, tibial extensor muscle activity, suggesting that spring-like properties of the leg and energy transfer from femur motion may play important roles in such leg movements. Adjacent legs sometime show coordinated femur movement during grooming with one hindleg, suggesting that grooming may also involve interlimb coordination.

Key words: scratching; motor control; insect; thoracic; ganglia; central pattern generation; locomotion
How do nervous systems control limb movements? One fruitful approach used throughout this century (Delcomyn, 1980) has involved the progressive elimination of parts of the nervous system to identify those that are crucial for limb motor control and to enable additional experiments on "simpler" and more convenient preparations. As a result of this approach, we know that the vertebrate spinal cord can produce basic patterns of limb motoneuron activity for locomotion and scratching through the action of local central pattern generator (CPG) circuits, i.e., in the absence of input from the brain and of movement-related sensory feedback (Gelfand et al., 1988; Stein, 1989). Experiments can now be focused on the central mechanisms of pattern generation as well as on the effects of specific brain and sensory inputs (Grillner and Dubuc, 1988; Rossignol et al., 1988; Pearson, 1993, 1995).

The neural control of limb movements in insects has arguably been less clear and more controversial than in vertebrates (Delcomyn, 1980; Pearson, 1985; Bässler, 1986, 1993; Cruse, 1990). Most studies have focused on the control of walking (see Graham, 1985). Roeder, for example, found that coordinated locomotion could occur in the praying mantis (Roeder, 1937) and the cockroach (Roeder, 1948) in the absence of the brain and subesophageal ganglion. The question of whether insect thoracic ganglia can produce appropriately coordinated leg movements in the absence

\footnotetext{
Received May 15, 1996; revised Aug. 27, 1996; accepted Sept. 20, 1996.

This research was supported by a National Research Service Award Postdoctoral Fellowship to A.B. and a National Science Foundation Presidential Faculty Fellowship to G.L. We thank two anonymous reviewers for helpful comments.

Correspondence should be addressed to Dr. Gilles Laurent, Division of Biology, 139-74, California Institute of Technology, Pasadena, CA 91125.

Dr. Berkowitz' current address: Department of Psychology, UCLA, Los Angeles, CA 90095-1563.

Copyright (C) 1996 Society for Neuroscience $0270-6474 / 96 / 168067-12 \$ 05.00 / 0$
}

of proprioceptive feedback, however, is still not settled (see Berkowitz and Laurent, 1996). A number of studies have demonstrated coordinated bursts of insect leg motoneuron activity in the absence of movement-related sensory feedback (Pearson and Iles, 1970, 1973; Pearson, 1972; Zilber-Gachelin and Chartier, 1973; Ryckebusch and Laurent, 1993, 1994; Büschges et al., 1995), but the links to actual leg movements have been uncertain (Reingold and Camhi, 1977; Sherman et al., 1977; Zill, 1986).

Here, we explore the neural control of leg movements in a behavior distinct from locomotion. Grooming (a behavior also known as scratching, cleaning, or wiping) involves directed limb movements and can be expressed in the absence of inputs from the brain in insects, amphibians, reptiles, and mammals (Rowell, 1961; Eaton and Farley, 1969; Vandervorst and Ghysen, 1980; Stein, 1983). In vertebrates, this behavior has the advantage that it can be reliably evoked by specific tactile stimuli, even in reduced preparations (Stein, 1983). By contrast, locomotor patterns in vertebrate-reduced preparations often require pharmacological or electrical stimulation (Gelfand et al., 1988). In addition, distinct forms of grooming can often be evoked by tactile stimulation of distinct regions of the body surface (Stein, 1983), allowing one to study the neural mechanisms of behavioral choice (Stein, 1989; Berkowitz and Stein, 1994a,b). The study of grooming may also help us understand the neural control of leg movements in insects. Locusts, cockroaches, and fruit flies can groom regions of the thorax or abdomen without input from the brain or subesophageal ganglion (Rowell, 1961; Eaton and Farley, 1969; Vandervorst and Ghysen, 1980). Much is already known about the anatomical and physiological organization of the locust thoracic nervous system (Burrows, 1992), providing an advantage for investigating the cellular control of grooming in this species. The large size of the 
locust hindleg also makes it especially suitable for movement analysis and muscle recording (Meyer, 1993). Moreover, if the locust thoracic ganglia can mediate grooming by more than one leg, one could examine the neural mechanisms of behavioral choice and potentially of interlimb coordination in this preparation as well.

To determine whether and how the locust thoracic ganglia can mediate grooming of multiple sites by more than one leg, we studied hindleg and middle leg site-specific grooming movements as well as simultaneously recorded hindleg electromyograms (EMGs) in locusts, the nerve cord of which was severed anterior to the mesothoracic ganglion. This work provides a foundation to address the issue of whether a thoracic CPG(s) can produce a rhythm(s) related to grooming. The companion paper (this issue) describes patterns of leg motoneuron activity during the same kinds of tactile stimulation in a similar preparation but in the absence of all leg motor innervation and, therefore, in the absence of movement-related sensory feedback.

Some of these data have been presented in abstract form (Berkowitz and Laurent, 1995).

\section{MATERIALS AND METHODS}

Preparation. All experiments were performed on adult male locusts, Schistocerca americana $(n=11)$, from a crowded laboratory colony. The connectives between the prothoracic and mesothoracic ganglia were exposed by cutting a small flap in the ventral cuticle and were severed with fine iridectomy scissors. The cuticle flap was then replaced and sealed over with melted wax. A segment of toothpick or thick insect pin was glued to the dorsal pronotum or the head using cyanoacrylate ester adhesive. The animal was suspended in midair, dorsal side up, by holding the toothpick or pin in a clamp. To prevent adjacent legs from removing EMG wires in EMG experiments, the contralateral hindleg was induced to autotomize, and the ipsilateral middle leg (and sometimes the ipsilateral front leg) was amputated at the trochanter and the stump sealed over with melted wax. In all cases, grooming was evoked by rubbing the tip of a fine paintbrush (3/0) continuously back and forth across a $2-5 \mathrm{~mm}$ region of the body surface at $1-4 \mathrm{~Hz}$ for as long as $1 \mathrm{~min}$ at a time; several minutes were allowed between stimulation episodes to avoid habituation. There was no relationship between the frequency with which the paintbrush was rubbed and the frequency of grooming leg movements; also, grooming sometimes continued for several cycles after removal of the paintbrush.

Movement analysis. Locusts were illuminated with floodlights and videotaped at $30 \mathrm{frames} / \mathrm{sec}$ via two genlocked (synchronized) video cameras (two Sanyo VDC2624 black and white CCD cameras or a Panasonic AG450 camcorder and a Panasonic WV-CL700 CCD camera) equipped with zoom lenses (Rainbow II and Computar Macrozoom) positioned on tripods at the height of the animal and facing the animal's side, $\sim 90^{\circ}$ apart. Recordings were made onto VHS videotapes (Fuji) using a Panasonic AG450 camcorder and a Panasonic AG7300 videocassette recorder. Videotapes were viewed on a Sony Trinitron video monitor. Peak Performance Technologies (Englewood, $\mathrm{CO}$ ) software was used to digitize the positions of selected locations on the animal during selected videotaped grooming movements from each camera view and to calculate the three-dimensional coordinates of these points as well as particular joint angles. Each video frame is composed of two sequential pictures, one made up of the odd lines of pixels and the other of the even lines. Every picture was digitized for each grooming episode, providing movement data at $60 \mathrm{~Hz}$. Fifteen points (for the experiment with intact legs) or 12 points (for experiments without the middle leg) on the animal were selected for digitization (Fig. $1 A$ ) as well as the paintbrush tip. Stick figure sequences (Figs. $1 B, 2 A, 3 A, 4 A$ ) were produced using Peak software and plotted onto a Hewlett-Packard 7475A plotter. The tibial extension angle of the middle leg or hindleg was calculated as the angle between the coxa-trochanter joint (the trochanter-femur joint is not articulated), the femur-tibia joint, and the tibia-tarsus joint. The femoral elevation angle was calculated differently for the middle leg and the hindleg, because the locust middle leg is oriented approximately perpendicular to the long axis of the body, whereas the locust hindleg remains approximately parallel to the long axis of the body during most grooming movements. The middle leg femoral elevation angle was calculated as the angle between the femur's long axis and the horizontal plane passing through the coxa-trochanter joint, with positive values when the femurtibia joint was above the trochanter and negative values when below. The hindleg femoral elevation angle was calculated as the angle between (1) the posterior, ventral corner of the wings, (2) the coxa-trochanter joint, and (3) the femur-tibia joint projected onto a vertical plane that passed through the long axis of the body (which was defined by a line through the anterior, ventral corner of the prothorax and the posterior, ventral corner of the wings). Latency was defined as the interval between the onset of tactile stimulation and the beginning of the first cycle of the leg movement that moved the tibia-tarsus joint against or near the site of stimulation. The latencies given in Results are for all grooming episodes that were analyzed in detail, whether with or without EMGs.

EMGs. Pairs of $50 \mu \mathrm{m}$ stainless steel wires (California Fine Wire, Grover Beach, CA), insulated except at the tips, were inserted just beyond the hindleg cuticle through holes in the cuticle made 1-2 mm apart with a fine insect pin and anchored with cyanoacrylate ester adhesive. Tibial extensor, tibial flexor, trochanteral levator, and trochanteral depressor muscle activities were recorded by placing pairs of wires in the dorsal femur, lateral femur, dorsolateral coxa, and ventrolateral coxa, respectively. EMG locations were confirmed by eliciting avoidance and resistance reflexes and listening to EMG signals on an audio monitor. Signals were amplified $(1000 \times)$ and filtered $(0.1-1 \mathrm{kHz}$ bandpass) using a differential AC amplifier (A-M Systems, Everett, WA). EMGs were digitized at $3 \mathrm{kHz}$ and synchronized with videotapes on-line using Peak Performance Technologies hardware and software. For determination of which began first on each cycle of grooming (the movement of a leg segment or activity in the corresponding muscle) when the movement and the EMG activity began at the same time, the cycle was counted as muscle first.

Phase histograms. The beginning of each cycle of grooming was defined as a local minimum in the femoral elevation angle unless changes in femoral elevation were too slight or irregular, in which case it was defined as a local minimum in the tibial extension angle. The joint angle used as the reference is indicated by the $x$-axis drawn below the corresponding phase histogram. The end of each cycle was defined as the data point immediately before $(1 / 60 \mathrm{sec}$ before for joint angles and $1 / 3000 \mathrm{sec}$ before for EMGs) the next local minimum. For joint angles, the set of angle values and phases for each cycle was fitted to a standard phase $x$-axis by cubic spline interpolation using Matlab (The MathWorks, Natick, MA). These normalized values were then averaged across all cycles of grooming in an episode. For EMGs, the absolute values of EMG voltage measurements were averaged within each of 20 bins (each bin being 1/20th of the cycle period) for each cycle. These averages were then averaged across all cycles of grooming in an episode. Thus, the $y$-axis values in EMG phase histograms are not inherently meaningful; only changes in the EMG $y$-axis values are important.

\section{RESULTS \\ Description of hindleg and middle leg grooming movements}

A locust with the connectives severed between the prothoracic and mesothoracic ganglia but with all the legs intact responded to tactile stimulation of at least four sites on the body surface by precisely moving the tibia-tarsus joint of the ipsilateral hindleg or middle leg against or near the site of stimulation (Figs. $1 B, 2 A$, $3 A, C, 4 A)$. The ipsilateral hindleg was used to groom the posterior abdomen (Fig. 1), the ventral or posterior side of the hindleg coxa (Fig. 2), and the ear (Fig. 3), which is located above the hindleg coxa. The ipsilateral middle leg was used to groom the anterior side of the hindleg coxa (Fig. 4). The tibia-tarsus joint was generally passed close to the site of stimulation, with or without contacting the body surface. The grooming leg occasionally contacted the paintbrush with sufficient force to push it away and end the stimulation [Fig. 3B,D (filled diamond)]. For the episodes of grooming analyzed in detail, the latency from tactile stimulus onset to grooming onset ranged from 0.4 to $26.0 \mathrm{sec}$ $($ mean $\pm \mathrm{SE}=5.1 \pm 7.8 \mathrm{sec})$ and was $<2 \mathrm{sec}$ in 8 of the 11 episodes. Grooming could also occur during tactile stimulation of other sites on the thorax and abdomen but with much less reli- 


\section{A Digitized Locations}

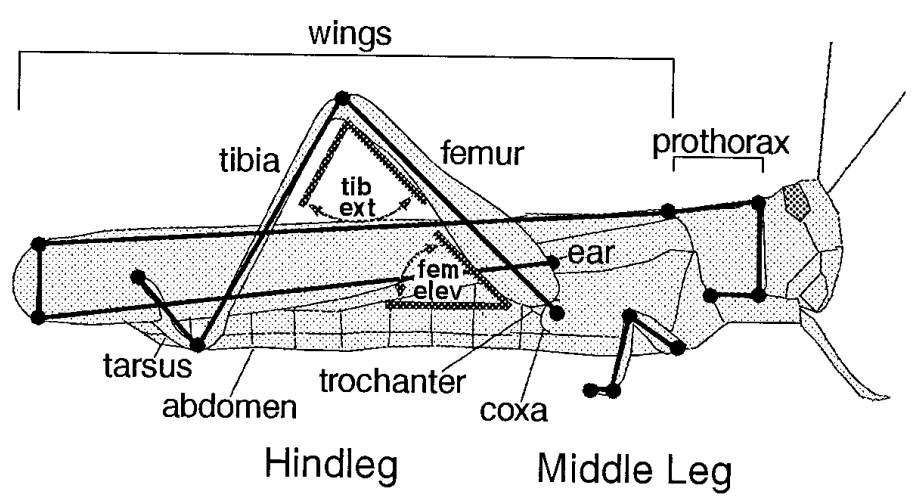

\section{Grooming of Posterior Abdomen}

\section{B Movement Sequence}

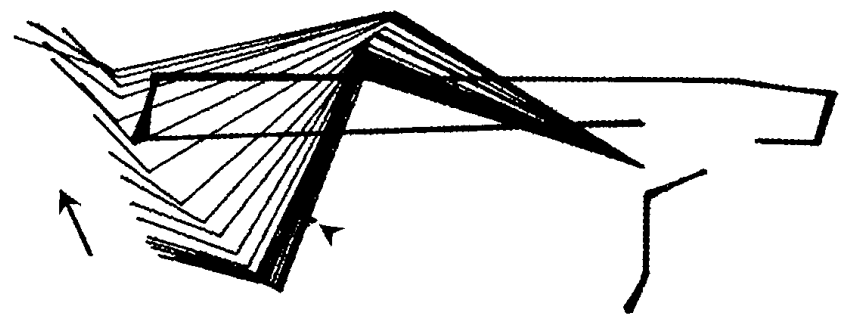

c Joint Angles
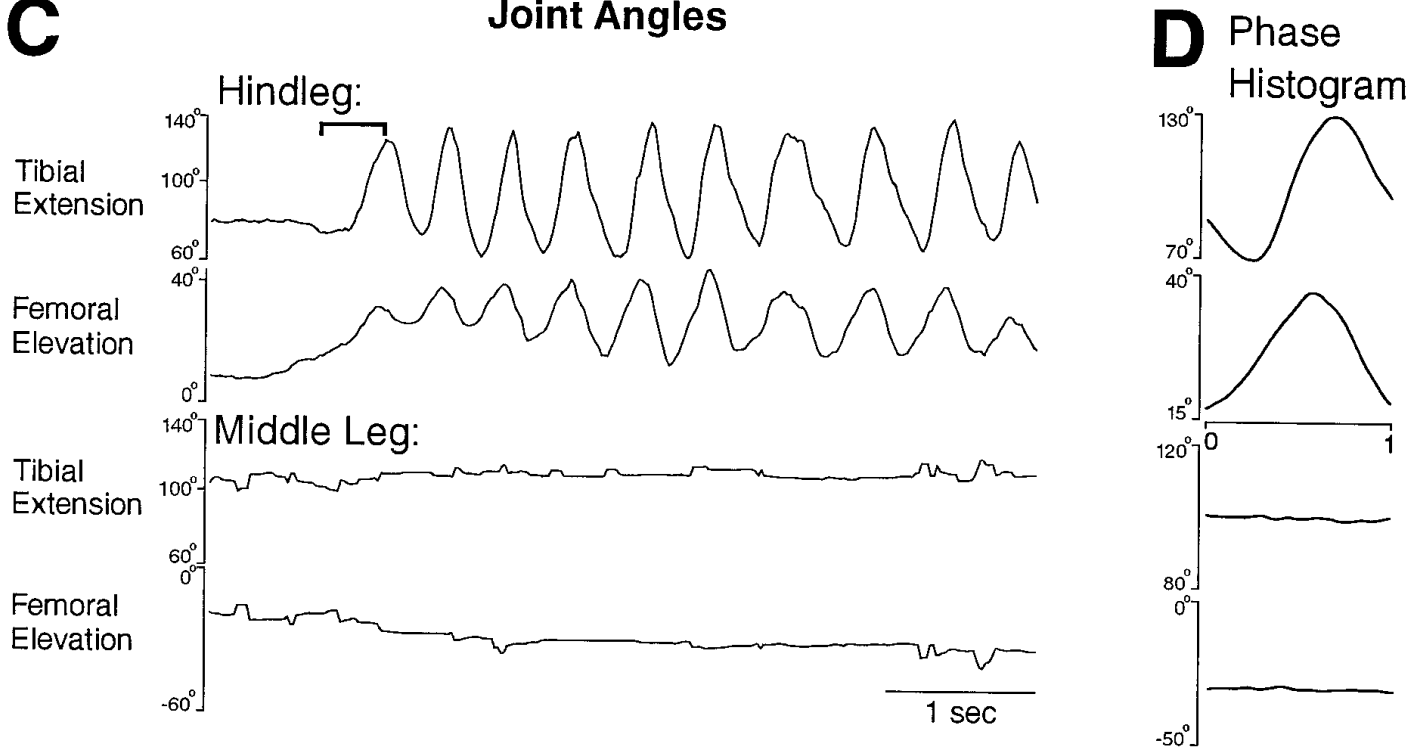

Figure 1. Hindleg grooming of the posterior abdomen with all legs intact. $A$, Schematic illustration of how stick figure sequences were generated from pairs of video images using 15 digitized locations on the right hindleg, middle leg, thorax, and wings. Each filled circle represents a digitized location. Joint angles measured are indicated in gray, for the hindleg only. tib ext, Tibial extension; fem elev, femoral elevation. $B$, Stick figure sequence for the time period indicated by the bracket in $C$. Arrow indicates the direction of leg movements. In addition to the 15 locations on the locust, the location of the stimulus site (on the abdomen) is indicated by a sequence of dots (arrowhead just above and anterior to the tibia-tarsus joint). $C$, Hindleg and middle leg tibial and femoral joint angles as a function of time during an episode of grooming of the posterior abdomen. Tactile stimulation was begun before the period shown and was continued throughout this period. See Materials and Methods for definitions of joint angles. $D$, Phase histogram of joint angles for the episode of grooming shown in $C$, generated from nine cycles. Each phase histogram plot is for the joint angle adjacent to it in $C$. The reference joint angle, in this case hindleg femoral elevation, is identified by placement of the phase $x$-axis under that plot. See Materials and Methods for method of calculating phase histograms.

ability; those types of grooming were not studied systematically or quantitatively.

Grooming often occurred as a sequence of regular rhythmic movements for hindleg grooming of the posterior abdomen (Fig.
$1 C$ ) or the ventral hindleg coxa (Fig. $2 B$ ) and for middle leg grooming of the anterior hindleg coxa (Fig. $4 B$ ), although sometimes only one cycle occurred. During each cycle of the rhythmic hindleg movements, the femur was lowered and raised, and the 


\section{Grooming of Ventral Hindleg Coxa}
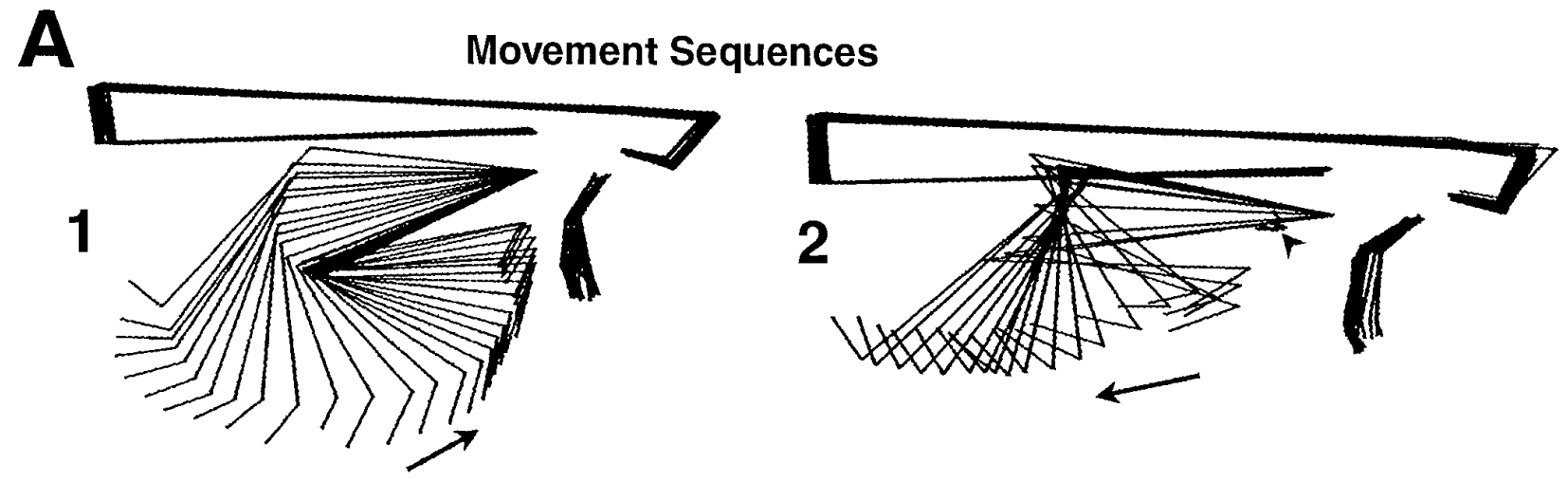

B
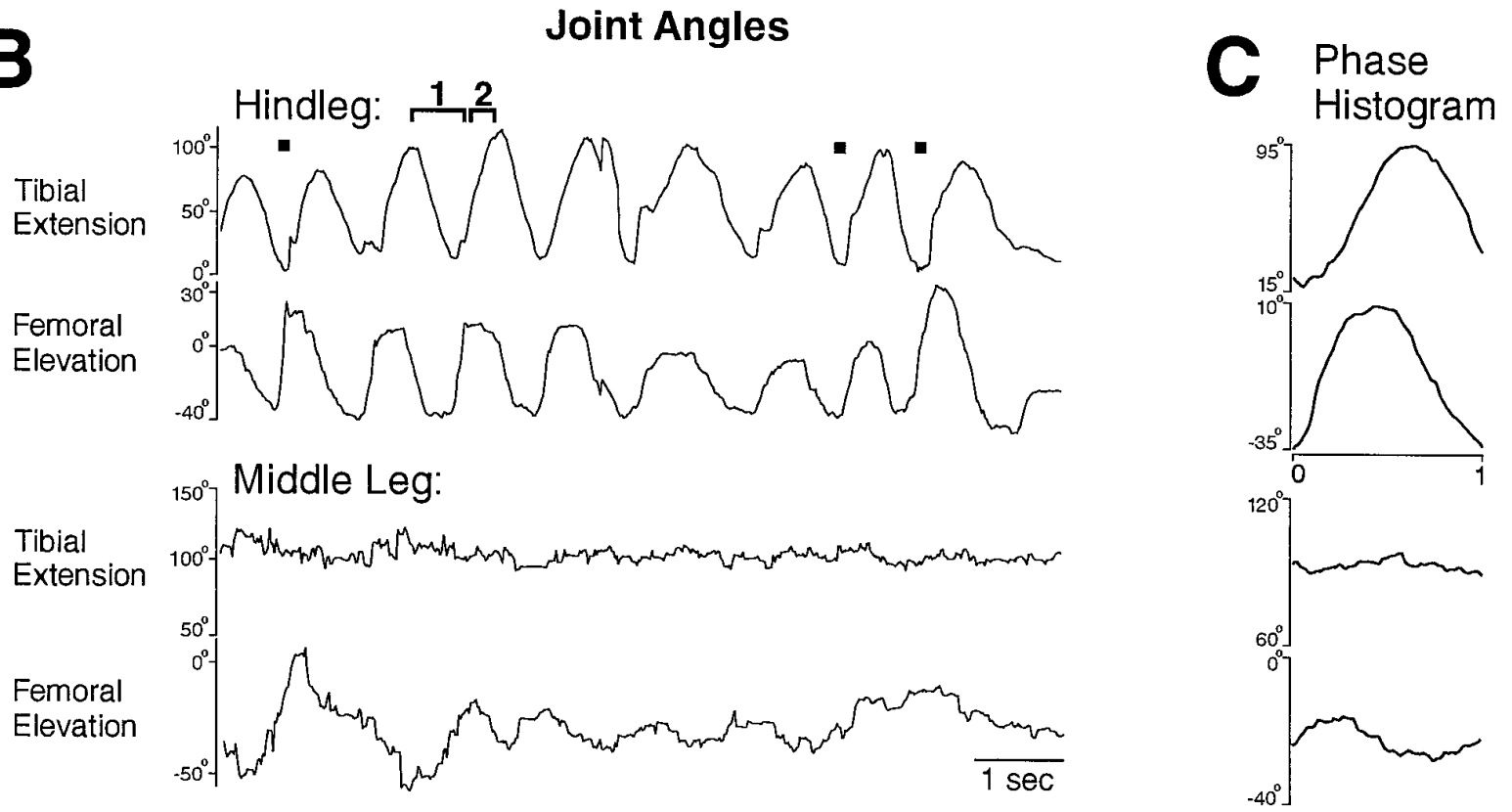

Figure 2. Hindleg grooming of the posterior ventral hindleg coxa (arrowhead) with all legs intact. $A, 1,2$, Stick figure sequences for two portions of one cycle of grooming for the time periods indicated by the brackets in $B . B$. Hindleg and middle leg tibial and femoral joint angles as a function of time during an episode of grooming of the posterior ventral hindleg coxa. Filled squares indicate contact between the hindleg tibia-tarsus joint and the site of stimulation. $C$, Phase histogram of joint angles for the episode of grooming shown in $B$, generated from eight cycles. The reference joint angle was hindleg femoral elevation. This is the same animal as shown in Figure 1; other conventions as in Figure 1.

tibia was flexed and extended (Figs. 1C, 2B). The tarsus was also rhythmically flexed and extended (data not shown). During each cycle of the rhythmic middle leg movements, the femur was lowered and raised but usually without large, regular movements at the femur-tibia joint (Fig. 4B). Hindleg grooming of the ear usually occurred one cycle at a time (Fig. 3), but multiple cycles of both femoral depression/elevation and tibial flexion/extension were sometimes observed (data not shown).

Hindleg grooming movements of the three selected sites were distinguished by the amplitudes of femoral elevation and depression. The hindleg femur, which is held just slightly above the horizontal in the undisturbed locust (data not shown) was raised to $30-40^{\circ}$ elevation during grooming of the posterior abdomen (Fig. 1) but was lowered to -30 to $-40^{\circ}$ during grooming of the ventral hindleg coxa (Fig. 2). During grooming of the ear, the hindleg femur was raised much farther than for the other sites, well beyond $90^{\circ}$ elevation (Fig. 3). In addition, the cycle period was approximately half as long for grooming of the posterior abdomen as for grooming of the ventral hindleg coxa (Figs. 1, 2).

The phase relationships between tibial extension and femoral elevation were quite similar for hindleg grooming of the posterior abdomen and the ventral hindleg coxa. In both cases, cycles of tibial extension and femoral elevation were coupled 1:1 and were nearly in-phase (Figs. 1D, 2C); femoral elevation led tibial extension slightly but consistently. In contrast, during hindleg grooming of the ear, tibial extension often did not begin until nearly the end of femoral elevation and the onset of femoral depression (Fig. $3 B, D)$. Tibial extension occurred smoothly and relatively slowly during grooming of the posterior abdomen (Fig. $1 B$ ) or ventral hindleg coxa (Fig. $2 A$ ), consistent with activity exclusively in the slow tibial extensor motoneuron. Large and sudden extensions of the tibia, however, could occur during ear grooming (Fig. 3C), suggesting activity of the fast tibial extensor motoneuron.

In general, the middle leg was relatively still during grooming 

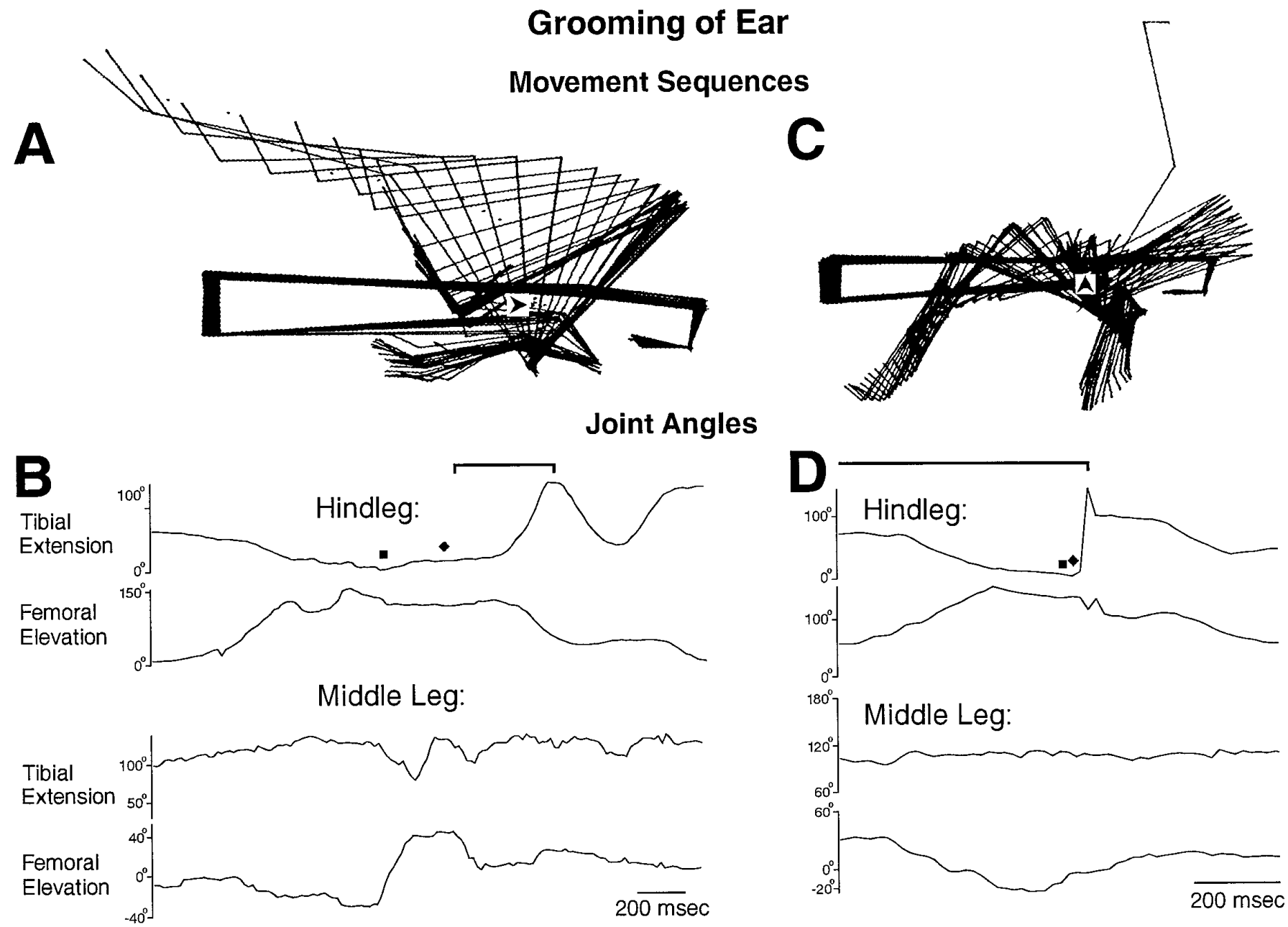

Figure 3. Hindleg grooming of the ear (located above the hindleg coxa) with all legs intact. $A, C$, Stick figure sequences for the time periods indicated by the brackets in $B$ and $D$, respectively. $B, D$, Hindleg and middle leg tibial and femoral joint angles as a function of time during the two episodes of grooming of the ear shown in $A$ and $C$. Filled square indicates contact between the hindleg tibia-tarsus joint and the site of stimulation; filled diamond indicates removal of the paintbrush from the stimulus site by the hindleg tibia-tarsus joint. This is the same animal as shown in Figures 1 and 2; other conventions as in Figures 1 and 2.

movements of the hindleg (Figs. 1-3), and the hindleg was relatively still during grooming movements of the middle leg, even if the site of stimulation was metathoracic (Fig. 4). In some cases, however, correlated movements of two ipsilateral adjacent legs were observed, suggesting interlimb coordination. In Figure $2 B$, for example, the middle leg femur was elevated in-phase with the hindleg femur on the first full cycle of grooming of the ventral hindleg coxa. In Figure $3 D$, the middle leg femur was depressed in-phase with the hindleg femur elevation during grooming of the ear. In addition, during all types of hindleg grooming, the contralateral hindleg was often raised or lowered in antiphase with the ipsilateral hindleg (data not shown); during grooming of the posterior abdomen, both hindlegs sometimes moved in phase to rub against this midline stimulation site.

Movements were relatively consistent from cycle to cycle and from one stimulation episode to another during hindleg grooming of the posterior abdomen (Fig. $5 A$ ) and the ventral hindleg coxa (Fig. $5 B$ ) and during middle leg grooming of the anterior hindleg coxa (Fig. $5 D$ ) but were more variable during hindleg grooming of the ear (Fig. 5C). Hindleg movements could include substantial femoral abduction as well as elevation during ear grooming (data not shown). Each of the three types of hindleg grooming occurred within a distinct region of femur-tibia "joint angle space" (Fig. $5 C, E)$, indicating that each movement was the result of a sitespecific motor strategy.

\section{Muscle activity during hindleg grooming movements}

In a second group of locusts $(n=10)$, the connectives of which were also severed between the prothoracic and mesothoracic ganglia, EMGs were recorded from hindleg tibial extensor and flexor and trochanteral levator and depressor muscle fibers while grooming movements of the hindleg were videotaped. (Trochanteral levator muscles raise, whereas trochanteral depressor muscles lower the femur.) The contralateral hindleg, the ipsilateral middle leg, and sometimes, the ipsilateral front leg were removed to prevent these legs from contacting or removing the hindleg EMG wires.

These animals produced hindleg grooming movements that were essentially the same as those produced by animals with all legs intact (compare Fig. 5, $C$ and $D$, and $E$ and $F$; Figs. 6 and 1; Figs. 7 and 2; and Figs. 8 and 3). Figures $6-8$ show hindleg movements and muscle activities for grooming of the posterior 


\section{Grooming of Anterior Hindleg Coxa}

\section{A Movement Sequence}
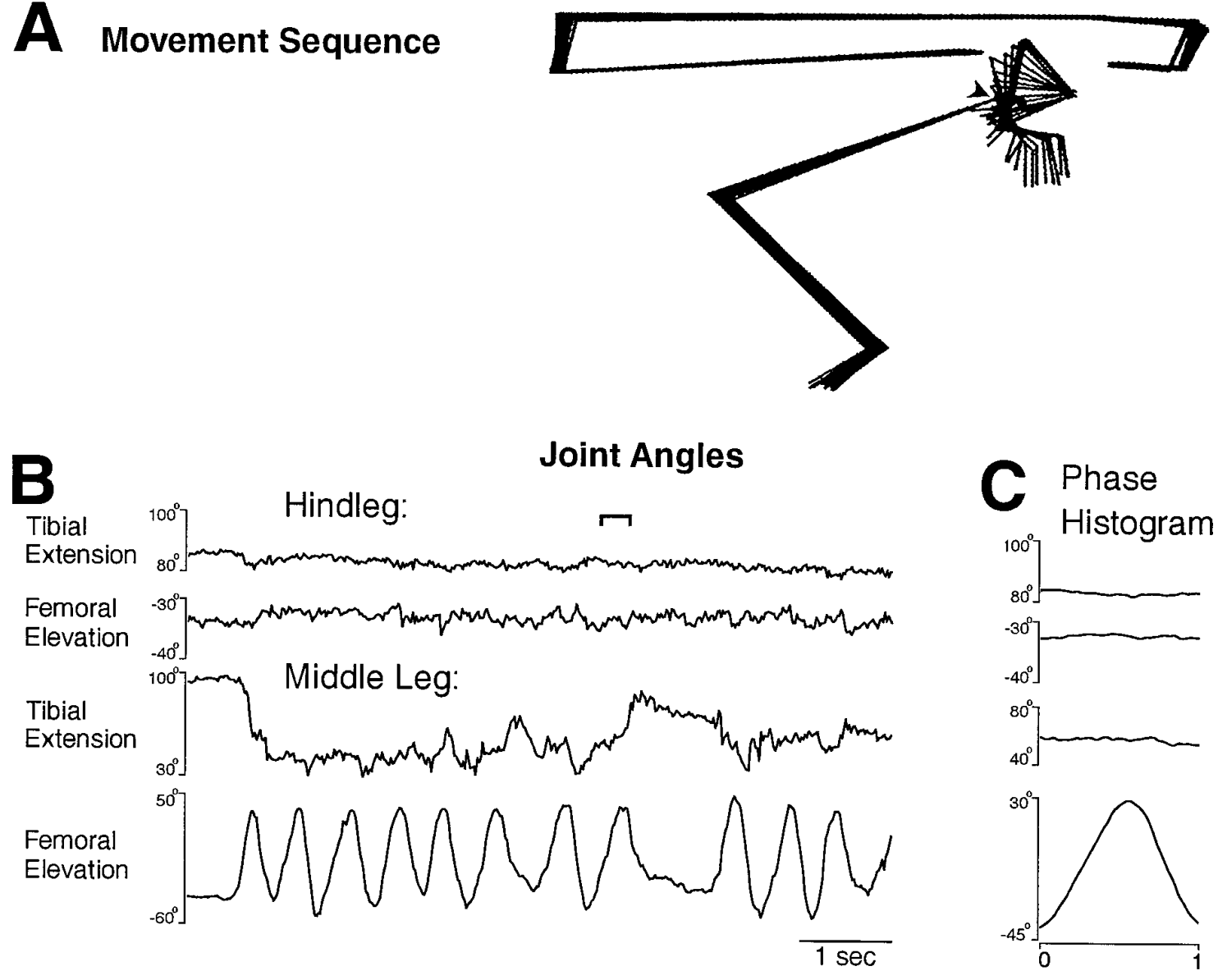

Figure 4. Middle leg grooming of the anterior hindleg coxa. A, Stick figure sequence for the time period indicated by the bracket in $B . B$, Hindleg and middle leg tibial and femoral joint angles as a function of time during an episode of grooming of the anterior hindleg coxa. $C$, Phase histogram of joint angles for the episode of grooming shown in $B$, generated from 12 cycles. The reference joint angle was middle leg femoral elevation. This is the same animal as shown in Figures 1-3.

abdomen, ventral hindleg coxa, and ear, respectively, in three different animals. Figure 9 shows hindleg movements and muscle activities for grooming of the same three sites in a single, different animal.

Hindleg grooming of the posterior abdomen (Figs. 6, 9A,B) or the ventral hindleg coxa (Figs. 7, 9C,D) often occurred as a sequence of regular rhythmic movements, during which each of the four muscles recorded exhibited rhythmic bursting activity. Bursts of the tibial extensor and tibial flexor muscles alternated as did bursts of the trochanteral levator and trochanteral depressor muscles. Bursts of the tibial extensor and trochanteral levator muscles largely overlapped, but in each cycle, the trochanteral levator burst began slightly before the tibial extensor burst. Tibial flexor and trochanteral depressor bursts also usually overlapped, but each trochanteral depressor burst began before the tibial flexor burst. Thus, for hindleg grooming of the posterior abdomen or ventral hindleg coxa, these four muscles were active in four distinct phases of grooming, but their bursts were coordinated 1:1 and were relatively phase-locked. The frequency of the grooming rhythm could slow down substantially toward the end of an episode, but the phase relationships among the muscles remained substantially the same (Fig. 6).

By contrast, when locusts made a sequence of hindleg grooming movements to the ear, tibial flexion/extension movements and muscle activities were no longer coupled 1:1 with trochanteral levator/depressor movements and muscle activities (Figs. 8, $9 E, F)$. Tibial extensor and flexor muscles were active in alternating bursts as for the other types of hindleg grooming (Figs. 8, $9 E, F)$. Before the hindleg was sufficiently elevated to bring the tibia-tarsus joint near the ear, the trochanteral levator and depressor muscles were sometimes active in alternating bursts, and the hindleg femur was raised and lowered moderately (Fig. 9E). The tibia-tarsus joint was brought to the ear when a long, large burst of trochanteral levator activity occurred and the femur was raised $>90^{\circ}$ (Figs. 8, 9E). Weaker coactivity of the trochanteral depressor also occurred at this time (Figs. 8, 9E). Thereafter, trochanteral levator and depressor muscles were largely coactive, primarily in bursts (Figs. 8, 9E,F). These bursts of levatordepressor coactivity occurred at a substantially lower frequency than the alternating bursts of the tibial extensor and flexor, so that multiple cycles of tibial extensor/flexor bursts occurred during each trochanteral levator-depressor cycle (Figs. 8, 9E,F). Correspondingly, multiple cycles of tibial extension/flexion occurred during each cycle of femoral elevation/depression (Figs. 8, 9E,F). An additional depressor burst sometimes occurred between leva- 

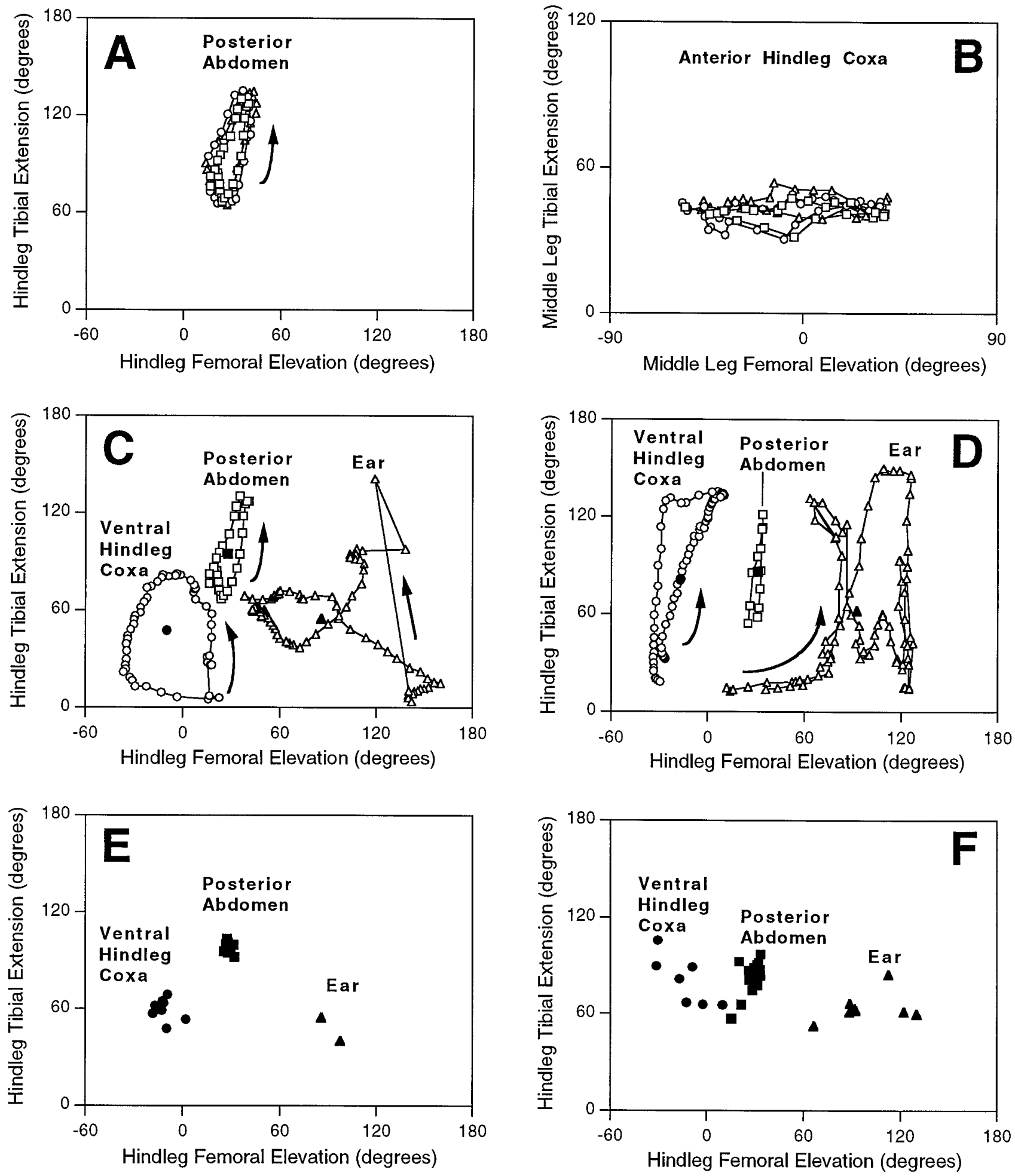

Figure 5. Trajectories in "joint angle space" during grooming of each site for animal with all legs intact $(A-C, E)$ and for animals in which EMGs were implanted in one hindleg and adjacent legs were removed $(D, F) . A-D$, Each open symbol indicates the femoral elevation angle $(x$-axis) and the tibial extension angle ( $y$-axis) calculated from one pair of digitized video images. Sequential open symbols are 1/60 sec apart. Arrows indicate the sequence of points when it was the same for all cycles shown. In $A$ and $C-F$, joint angles are for the hindleg; in $B$, joint angles are for the middle leg. $A, B$, Three consecutive cycles of grooming for hindleg grooming of the posterior abdomen and middle leg grooming of the anterior hindleg coxa from the episodes in Figures 1 and 4, respectively. In each case, open squares indicate the first cycle, open circles indicate the second cycle, and open triangles indicate the third cycle. $C, D$, Comparison of joint angle trajectories for grooming of the three sites by the hindleg. One cycle each is plotted from the episodes in Figures 1-3 (for $C$ ) and from the episodes in Figures 6, 7, and $9 E$ (for $D$ ), using open symbols (circle for ventral hindleg coxa; square for posterior abdomen; triangle for ear). The corresponding filled symbol in each case indicates the "center of mass" of the cycle, i.e., the mean femoral elevation and mean tibial extension values. $C$, All legs intact. $D$, EMG experiments. $E, F$, Center of mass of each cycle is plotted for all analyzed cycles of hindleg grooming of each site; symbols as in $C$ and $D$. E, All legs intact; $F$, EMG experiments. 


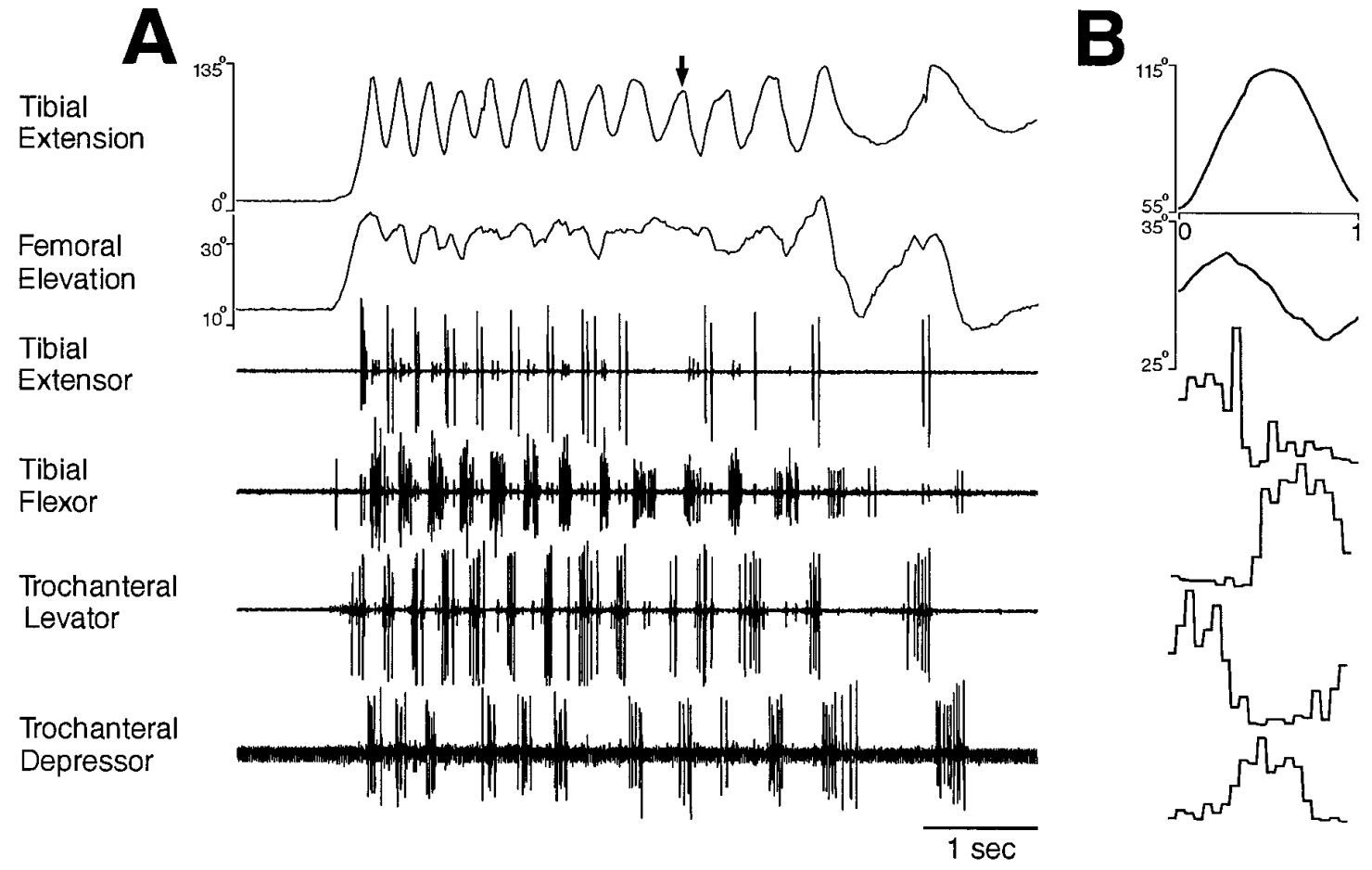

Figure 6. Hindleg joint angles and simultaneous hindleg EMG recordings for hindleg grooming of the posterior abdomen. From top to bottom, traces are tibial extension angle, femoral elevation angle, tibial extensor EMG, tibial flexor EMG, trochanteral levator EMG, and trochanteral depressor EMG. $A$, Joint angles and EMGs as a function of time. Arrow indicates a cycle of tibial extension that occurred without recorded tibial extensor muscle activity. $B$, Phase histogram of joint angles and EMGs for the episode of grooming shown in $A$, generated from 13 cycles. The reference joint angle was tibial extension.

tor-depressor bursts, the effect of which appeared to be a net femoral depression movement (Fig. 9E,F).

In general, the hindleg femur was raised when the trochanteral levator muscle was active and lowered when the trochanteral depressor muscle was active; the tibia was extended when the tibial extensor muscle was active and flexed when the tibial flexor muscle was active. There were, however, important exceptions to this rule. For all grooming episodes analyzed in detail, tibial extension began before tibial extensor muscle activity in $28(50 \%)$ of 56 grooming cycles; tibial flexion began before tibial flexor muscle activity in 15 (27\%) of 56 cycles, femoral elevation began before trochanteral levator muscle activity in 5 (14\%) of 36 cycles, and femoral depression began before trochanteral depressor muscle activity in $5(20 \%)$ of 25 cycles. Thus, for tibial extension in particular, the movement of the leg segment could begin before recorded activity in the corresponding muscle, even on the first cycle of a grooming episode when the hindleg had previously been still (Figs. 6, 9A). Tibial extension could even occur in the absence of any recorded tibial extensor muscle activity (Figs. 6 $A, 8 A$, $9 A, E$, arrows). In one case, this tibial extension carried enough force to push the paintbrush away [Fig. 9A, third cycle (filled diamond)].

\section{DISCUSSION}

Locusts were able to groom at least four sites on the thorax and abdomen using the hindleg or the middle leg without input from the brain, subesophageal ganglion, or prothoracic ganglion. Amputation of adjacent legs had little effect on hindleg grooming movements, in contrast to the effects of leg amputation on insect locomotion (see Graham, 1985). The movement analyses and simultaneous EMGs provided in this paper will provide a foun- dation for comparison with centrally generated leg motor patterns obtained in a similar paradigm in the companion paper. The bumpy history of studies on the neural control of insect locomotion suggests that a step-wise approach is advisable (Pearson and Iles, 1970; Pearson, 1972; Zilber-Gachelin and Chartier, 1973; Reingold and Camhi, 1977; Sherman et al., 1977; Pearson, 1985; Zill, 1986) (see also Berkowitz and Laurent, 1996).

The current description of middle leg and hindleg grooming adds to previous work that described locust front leg grooming (Rowell, 1961) and demonstrated that front leg grooming is facilitated by progressive isolation of the prothoracic ganglion from other ganglia (Rowell, 1964). The current results are consistent with a recent description of accurate grooming of thoracic sites in response to tactile stimulation in intact locusts (Meyer, 1993).

\section{Grooming and walking motor patterns are distinct}

During rhythmic hindleg grooming of the posterior abdomen or the ventral hindleg coxa, tibial extension occurred approximately in-phase with femoral elevation, and tibial extensor muscle activity occurred approximately in-phase with trochanteral levator muscle activity. These hindleg movements and motor patterns are distinct from those that occur during locust walking. When locusts walk, tibial extension occurs primarily during femoral depression, when the leg is in contact with the ground, i.e., during the stance phase (Burns and Usherwood, 1979; Graham, 1985). During pilocarpine-evoked rhythms in the isolated locust metathoracic ganglion, slow tibial extensor motoneuron bursts occur in-phase with trochanteral depressor motoneuron bursts (Ryckebusch and Laurent, 1993), a pattern similar to walking but distinct from grooming. 


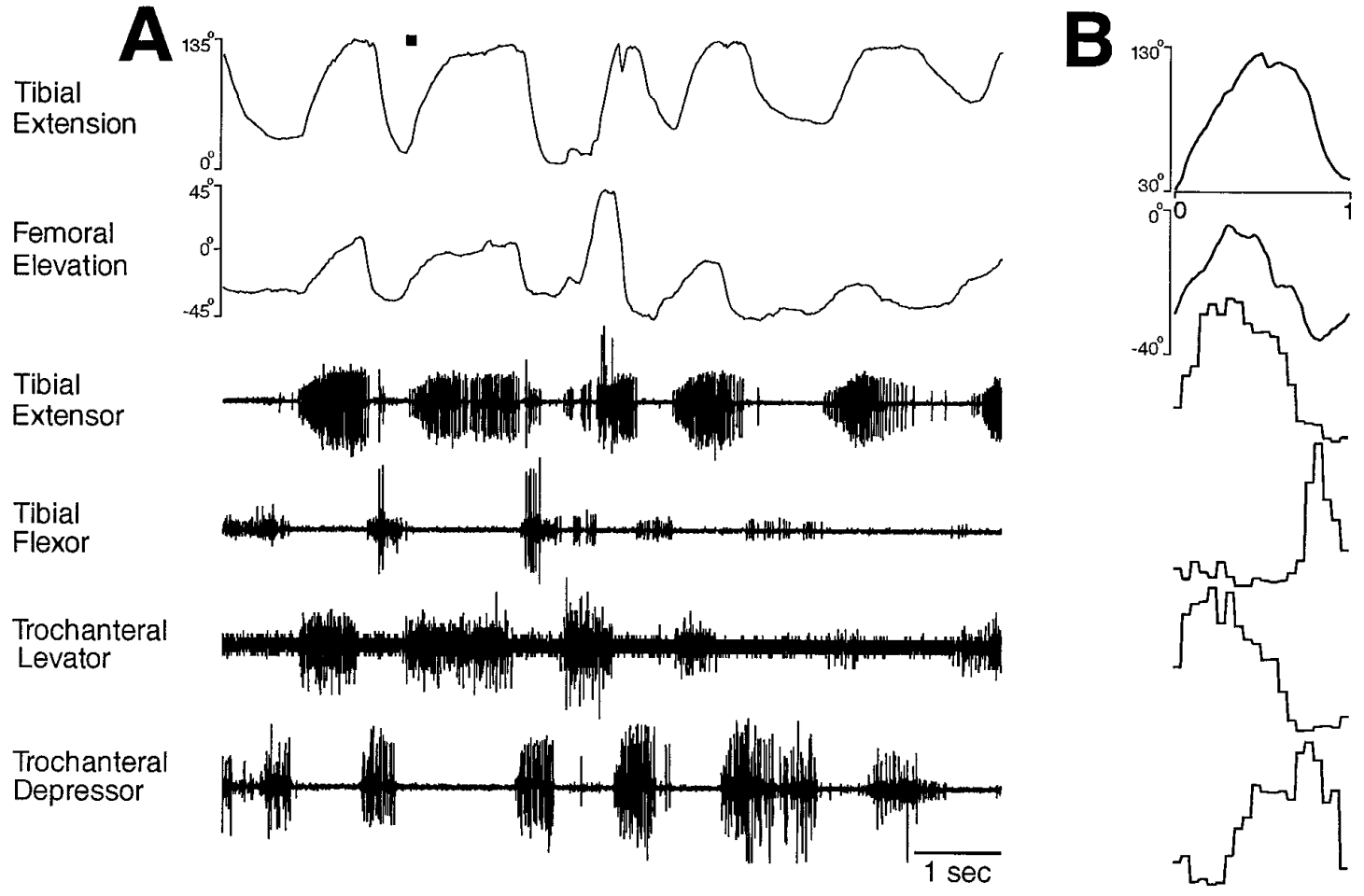

Figure 7. Hindleg joint angles and simultaneous hindleg EMG recordings for hindleg grooming of the ventral hindleg coxa. $A$, Joint angles and EMGs as a function of time. $B$, Phase histogram of joint angles and EMGs for the episode of grooming shown in $A$, generated from five cycles. The reference joint angle was tibial extension. These data were recorded from a different animal than the data in Figure 6.

\section{Dissociable modules control the femur and the tibia}

During hindleg grooming of the ear, multiple cycles of tibial extension/flexion could occur during each cycle of femoral elevation/depression, and multiple bursts of alternating tibial extensor and flexor muscle activity could occur during each burst of the trochanteral levator and depressor muscles. This demonstrates that the nervous system can decouple the activity of muscles that move the tibia and the femur, even though they are coupled 1:1

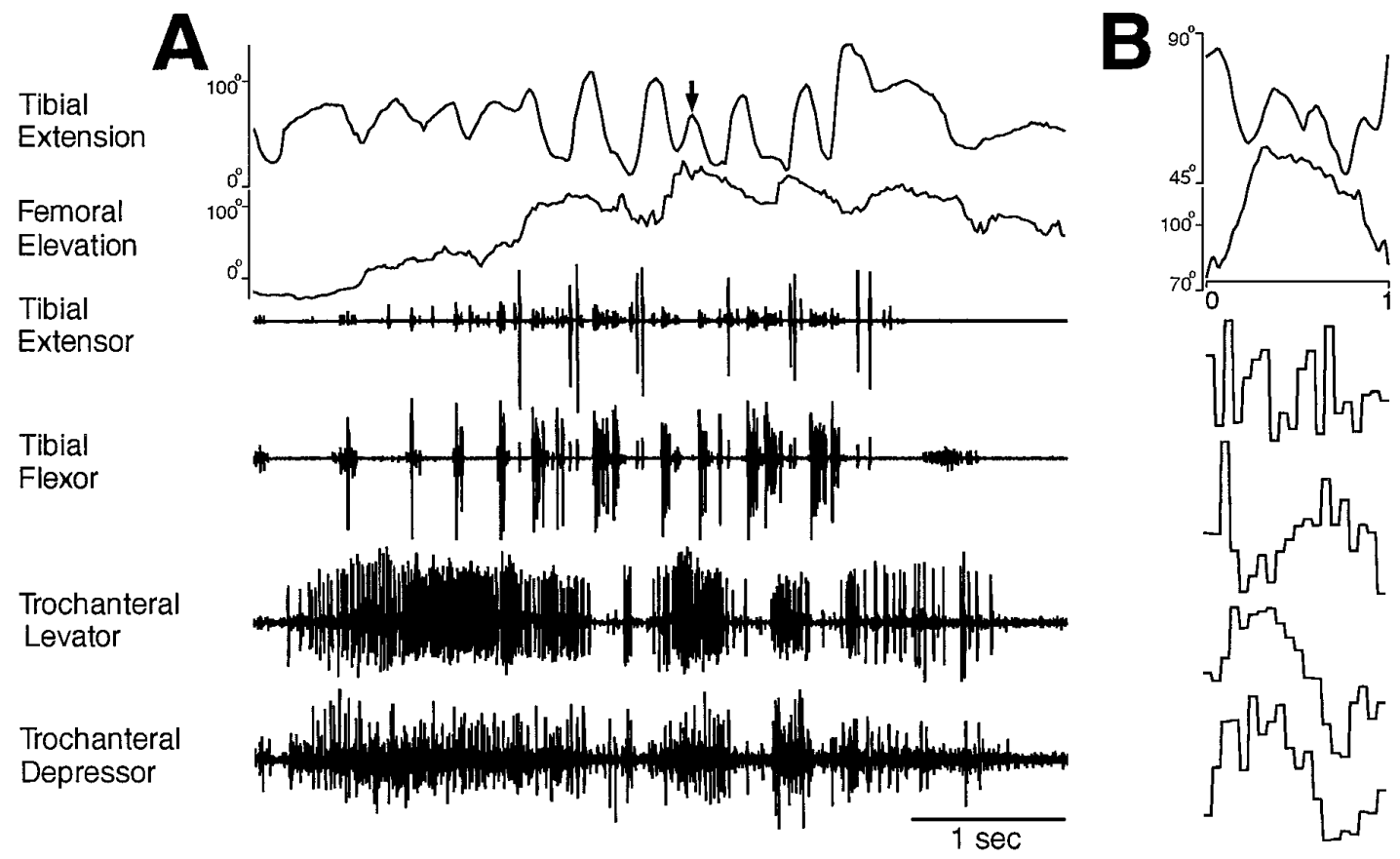

Figure 8. Hindleg joint angles and simultaneous hindleg EMG recordings for hindleg grooming of the ear. $A$, Joint angles and EMGs as a function of time. $B$, Phase histogram of joint angles and EMGs for the episode of grooming shown in $A$, generated from four cycles of femoral elevation. The reference joint angle was femoral elevation. These data were recorded from a different animal than the data in Figures 6 and 7. 

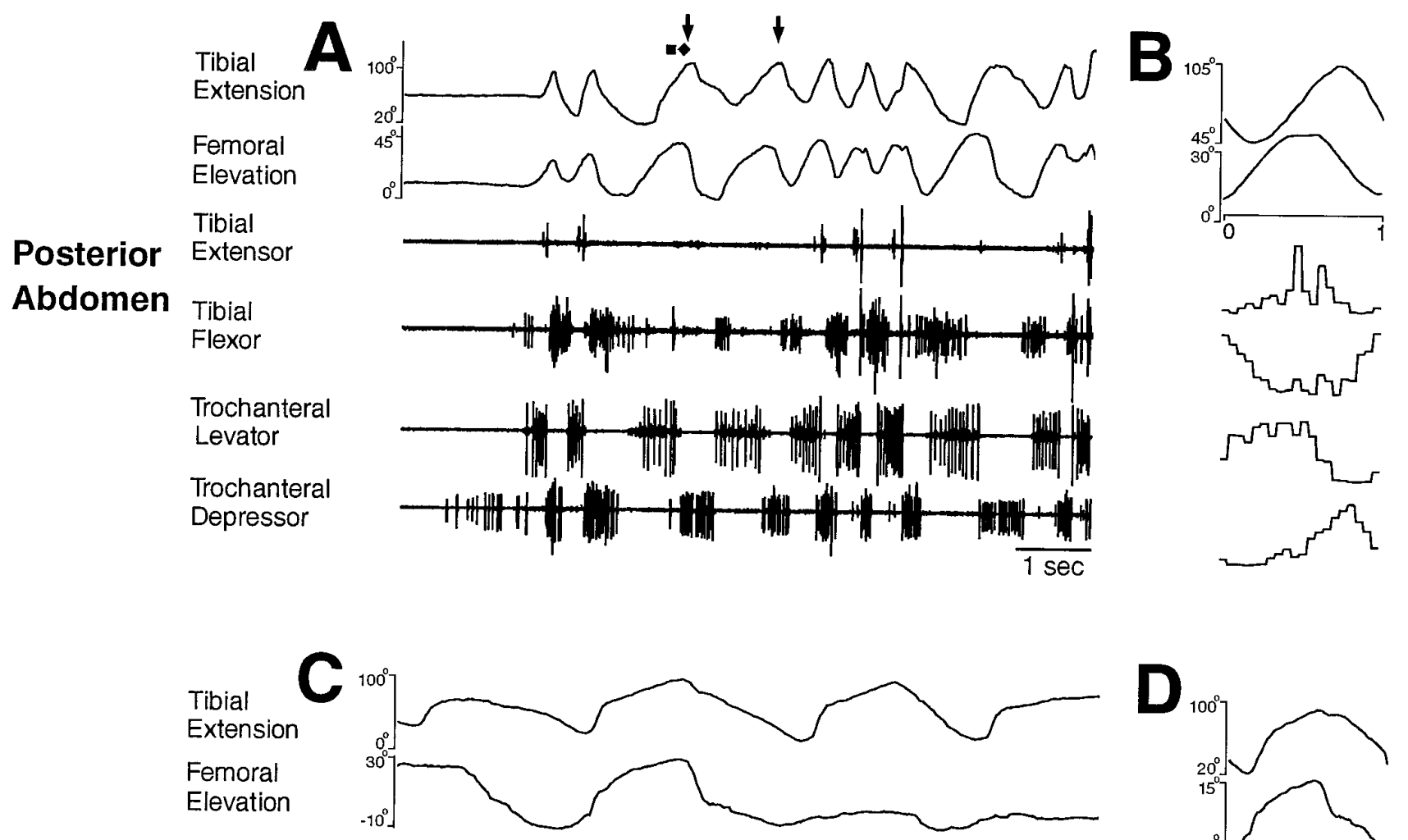

\section{Ventral Tibial Hindleg Extensor Coxa \\ Tibial \\ Flexor \\ Trochanteral Levator \\ Trochanteral Depressor}
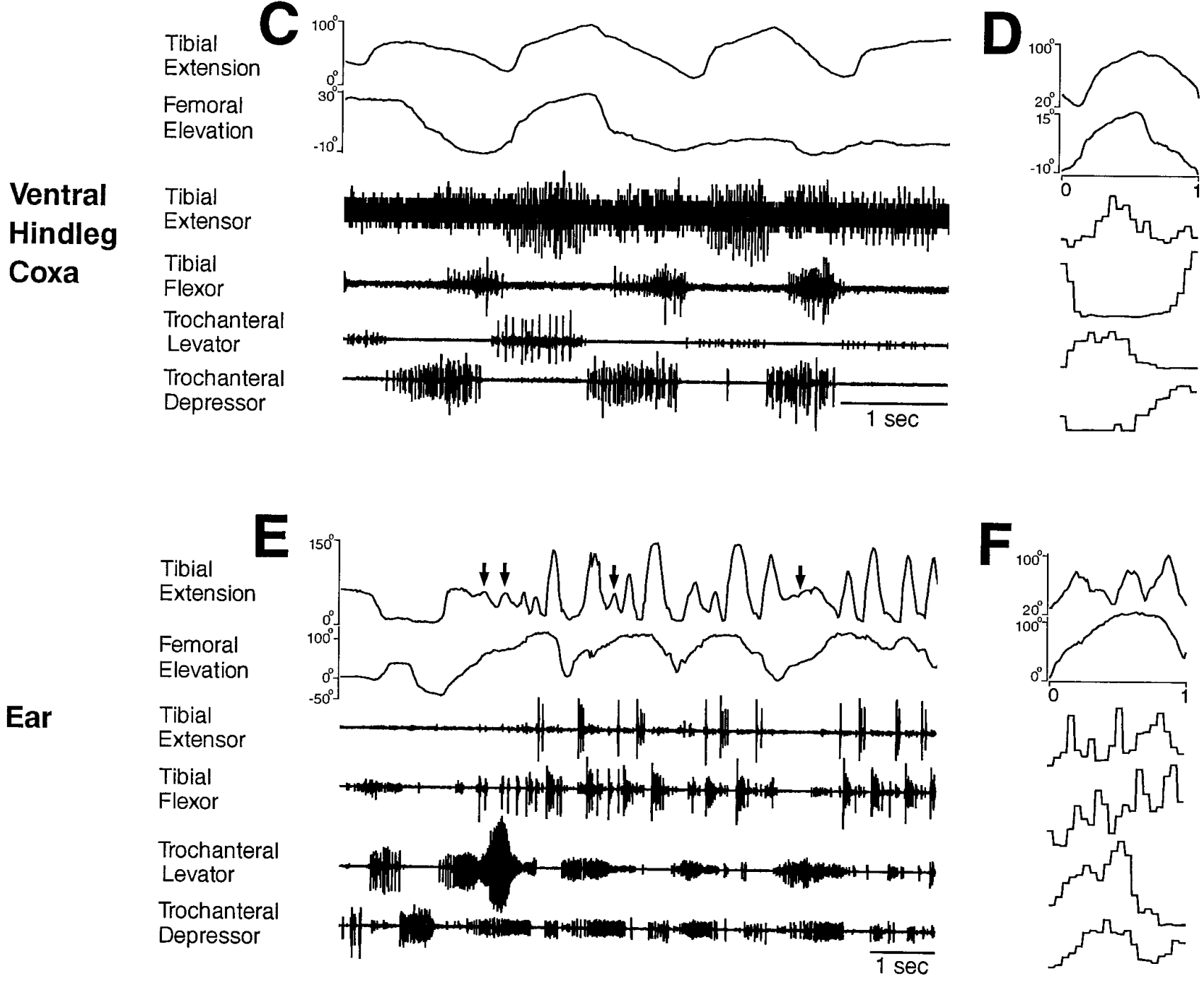

Figure 9. Hindleg joint angles and simultaneous hindleg EMG recordings for hindleg grooming of all three sites in a single animal. $A, B$, Posterior abdomen; $C, D$, ventral hindleg coxa; $E, F$, ear. $A, C, E$, Joint angles and EMGs as a function of time; $B, D, F$, phase histograms of joint angles and EMGs for the episodes of grooming shown in $A, C$, and $E$, respectively, generated from eight, two, and four cycles (of femoral elevation), respectively. The reference joint angle was femoral elevation in each case. For clarity, the gain of each joint angle and EMG trace has been adjusted to maximize the signal. These data were recorded from a different animal than the data in Figures 6-8. 
during other behaviors such as hindleg grooming of the posterior abdomen and the ventral hindleg coxa and hindleg walking movements (Burns and Usherwood, 1979; Graham, 1985). Evidence from the stick insect also suggests that femoral and tibial modules can be decoupled (Bässler, 1993; Büschges et al., 1995). These findings are consistent with Grillner's proposal (1981) that locomotor movements about each limb joint are controlled by a distinct "unit burst generator," although the generators for different joints of the same limb are usually coupled 1:1. Hindleg movements during walking and the three types of locust hindleg grooming might be generated by a single set of unit burst generators, with distinct coupling between tibial and femoral generators in (1) walking, (2) grooming of the posterior abdomen or ventral hindleg coxa, and (3) grooming of the ear. Distinct behaviors, such as digestive rhythms in crustaceans and swallowing, mastication, respiration, and stepping in mammals, can also be coupled and decoupled (Dickinson, 1995).

Grooming of the ear can also involve strong coactivation of trochanteral levators and depressors in contrast to both walking and the other types of grooming. This coactivation might depend on proprioceptive feedback (see Berkowitz and Laurent, 1996).

\section{Movements without muscle activity?}

Tibial extension often began before tibial extensor muscle activity during hindleg grooming, even on the first cycle of grooming. Occasionally, the tibial extensor muscle burst was missing for one or more cycles, and yet apparently normal tibial extension still occurred and could be strong enough to push away the paintbrush.

How does the hindleg tibia extend without tibial extensor muscle activity? One possible explanation involves a variety of "catchlike" mechanisms that can cause muscle tension even in the absence of activity in the motoneurons that innervate it (Hoyle, 1983). The locust tibial extensor muscle can exhibit catch-like tension (Evans and Siegler, 1982; Hoyle, 1983). This phenomenon, however, was observed during a maintained posture and not during rhythmic movements (Evans and Siegler, 1982; Hoyle, 1983). A modulatory motoneuron that innervates the locust hindleg tibial extensor muscle dramatically reduces this catch-like tension and is thought to be active during movements (Evans and Siegler, 1982). Thus, catch-like tension appears unlikely to play a major role during grooming.

Another explanation involves the mechanical properties of the cuticle, connective, and muscle tissues. For example, limb segments may spring back to an intermediate position determined partly by passive muscle tension (Yox et al., 1982) at the end of a period of muscle activation. In this way, tibial extension may begin as soon as the tibial flexor muscle burst has ceased, even if the tibial extensor muscle has not yet become active. In fact, when tibial extension occurred before tibial extensor muscle activity, even on the first cycle of grooming, brief tibial flexor muscle activity occurred just before tibial extension began. This springlike effect, however, appears unlikely to generate a normal tibial extension or enough force to push away the paintbrush (Fig. 9A). A different mechanical contribution may be energy transfer from elevation of the femur: as the femur is raised quickly, it may tend to "fling" the tibia into an extended position even in the absence of tibial extensor muscle activity. In fact, during normal extension of the tibia without tibial extensor muscle activity, a strong burst of the trochanteral levator muscle and elevation of the femur preceded tibial extension (Fig. 9A). These suggestions emphasize the importance of understanding limb mechanics if one is to understand the control of limb movements by the nervous system.
Our results also indicate that patterns of muscle or motoneuron activity may not be sufficient to predict movements of the limb they control.

\section{Interlimb coordination during grooming?}

This paper provides suggestive evidence that movements of a grooming limb may be coordinated with movements of an adjacent limb. During hindleg grooming, coordinated movements were sometimes seen in the ipsilateral middle leg or the contralateral hindleg (see Results). Recently, it has been shown that the turtle spinal cord generation of hindleg scratching, usually thought to be unilateral, relies partly on contralateral neural circuitry (Berkowitz and Stein, 1994a,b; Stein et al., 1995). Thus, there may be interlimb coordination in grooming, as in walking (Graham, 1985). If so, similar mechanisms of interlimb coordination might be used in these two behaviors. This raises the intriguing possibility that a locust preparation in which all leg motor innervation is severed might be used not only to test for centrally generated motor patterns used for grooming but also to test for centrally generated interlimb coordination (see also Ryckebusch and Laurent, 1994). This possibility is explored further in the companion paper.

\section{REFERENCES}

Bässler U (1986) On the definition of central pattern generator and its sensory control. Biol Cybern 54:65-69.

Bässler U (1993) The walking- (and searching-) pattern generator of stick insects, a modular system composed of reflex chains and endogenous oscillators. Biol Cybern 69:305-317.

Berkowitz A, Laurent G (1995) Central generation of directed limb movements in locusts. Soc Neurosci Abstr 21:1764.

Berkowitz A, Laurent G (1996) Central generation of grooming motor patterns and interlimb coordination in locusts. $\mathrm{J}$ Neurosci 16:8079-8091.

Berkowitz A, Stein PSG (1994a) Activity of descending propriospinal axons in the turtle hindlimb enlargement during two forms of fictive scratching: broad tuning to regions of the body surface. J Neurosci 14:5089-5104.

Berkowitz A, Stein PSG (1994b) Activity of descending propriospinal axons in the turtle hindlimb enlargement during two forms of fictive scratching: phase analyses. J Neurosci 14:5105-5119.

Burns MD, Usherwood PNR (1979) The control of walking in Orthoptera. II. Motor neurone activity in normal free-walking animals. J Exp Biol 79:69-98.

Burrows M (1992) Local circuits for the control of leg movements in an insect. Trends Neurosci 15:226-232.

Büschges A, Schmitz J, Bässler U (1995) Rhythmic patterns in the thoracic nerve cord of the stick insect induced by pilocarpine. J Exp Biol 198:435-456.

Cruse H (1990) What mechanisms coordinate leg movement in walking arthropods? Trends Neurosci 13:15-21.

Delcomyn F (1980) Neural basis of rhythmic behavior in animals. Science 210:492-498.

Dickinson PS (1995) Interaction among neural networks for behavior. Curr Opin Neurobiol 5:792-798.

Eaton RC, Farley RD (1969) The neural control of cercal grooming behaviour in the cockroach, Periplaneta americana. J Insect Physiol 15:1047-1065.

Evans PD, Siegler MVS (1982) Octopamine mediated relaxation of maintained and catch tension in locust skeletal muscle. J Physiol (Lond) 324:93-112.

Gelfand IM, Orlovsky GN, Shik ML (1988) Locomotion and scratching in tetrapods. In: Neural control of rhythmic movements in vertebrates (Cohen AH, Rossignol S, Grillner S, eds), pp 167-199. New York: Wiley.

Graham D (1985) Pattern and control of walking in insects. Adv Insect Physiol 18:31-140.

Grillner S (1981) Control of locomotion in bipeds, tetrapods, and fish. In: Handbook of physiology, Sec 1, The nervous system, Vol 2, Motor control (Brooks V, ed), pp 1179-1236. Bethesda, MD: American Physiological Society. 
Grillner S, Dubuc R (1988) Control of locomotion in vertebrates: spinal and supraspinal mechanisms. In: Functional recovery in neurological disease (Waxman SG, ed), pp 425-453. New York: Raven.

Hoyle G (1983) Forms of modulatable tension in skeletal muscles. Comp Biochem Physiol 76A:203-210.

Meyer TS (1993) Patterns of activity in metathoracic leg muscles during rhythmic scratching behaviour in the locust. Proceedings of the XXXII Congress of the International Union of Physiological Sciences 32:174.

Pearson KG (1972) Central programming and reflex control of walking in the cockroach. J Exp Biol 56:173-193.

Pearson KG (1985) Are there central pattern generators for walking and flight in insects? In: Feedback and motor control in invertebrates and vertebrates (Barnes WJP, ed), pp 307-315. London: Croom Helm.

Pearson KG (1993) Common principles of motor control in vertebrates and invertebrates. Annu Rev Neurosci 16:265-297.

Pearson KG (1995) Proprioceptive regulation of locomotion. Curr Opin Neurobiol 5:786-791.

Pearson KG, Iles JF (1970) Discharge patterns of coxal levator and depressor motoneurones of the cockroach, Periplaneta americana. J Exp Biol 52:139-165.

Pearson KG, Iles JF (1973) Nervous mechanisms underlying intersegmental co-ordination of leg movements during walking in the cockroach. J Exp Biol 58:725-744.

Reingold SC, Camhi JM (1977) A quantitative analysis of rhythmic leg movements during three different behaviors in the cockroach, Periplaneta americana. J Insect Physiol 23:1407-1420.

Roeder KD (1937) The control of tonus and locomotor activity in the praying mantis (Mantis religiosa L.). J Exp Zool 76:353-374.

Roeder KD (1948) Organization of the ascending giant fiber system in the cockroach (Periplaneta americana). J Exp Zool 108:243-261.

Rossignol S, Lund JP, Drew T (1988) The role of sensory inputs in regulating patterns of rhythmical movements in higher vertebrates. In: Neural control of rhythmic movements in vertebrates (Cohen $\mathrm{AH}$ Rossignol S, Grillner S, eds), pp 201-283. New York: Wiley.
Rowell CHF (1961) The structure and function of the prothoracic spine of the desert locust, Schistocerca gregaria Forskal. J Exp Biol 38:457-469.

Rowell CHF (1964) Central control of an insect segmental reflex. I. Inhibition by different parts of the central nervous system. J Exp Biol 41:559-572.

Ryckebusch S, Laurent G (1993) Rhythmic patterns evoked in locust leg motor neurons by the muscarinic agonist pilocarpine. J Neurophysiol 69:1583-1595.

Ryckebusch S, Laurent G (1994) Interactions between segmental leg central pattern generators during fictive rhythms in the locust. J Neurophysiol 72:2771-2785.

Sherman E, Novotny M, Camhi JM (1977) A modified walking rhythm employed during righting behavior in the cockroach Gromphadorhina portentosa. J Comp Physiol [A] 113:303-316.

Stein PSG (1983) The vertebrate scratch reflex. Symp Soc Exp Biol 37:383-403.

Stein PSG (1989) Spinal cord circuits for motor pattern selection in the turtle. Ann NY Acad Sci 563:1-10.

Stein PSG, Victor JC, Field EC, Currie SN (1995) Bilateral control of hindlimb scratching in the spinal turtle: contralateral spinal circuitry contributes to the normal ipsilateral motor pattern of fictive rostral scratching. J Neurosci 15:4343-4355.

Vandervorst P, Ghysen A (1980) Genetic control of sensory connections in Drosophila. Nature 286:65-67.

Yox DP, DiCaprio RA, Fourtner CR (1982) Resting tension and posture in arthropods. J Exp Biol 96:421-425.

Zilber-Gachelin NF, Chartier MP (1973) Modification of the motor reflex responses due to repetition of the peripheral stimulus in the cockroach. II. Conditions of activation of the motoneurones. J Exp Biol 59:383-403.

Zill SN (1986) A model of pattern generation of cockroach walking reconsidered. J Neurobiol 17:317-328. 